\title{
RIGOROUS MULTIPLICATIVE PERTURBATION BOUNDS FOR THE GENERALIZED CHOLESKY FACTORIZATION AND THE CHOLESKY-LIKE FACTORIZATION
}

\author{
HANYU LI AND YANFEI YANG
}

\begin{abstract}
The generalized Cholesky factorization and the Cholesky-like factorization are two generalizations of the classic Cholesky factorization. In this paper, the rigorous multiplicative perturbation bounds for the two factorizations are derived using the matrix equation and the refined matrix equation approaches. The corresponding first-order multiplicative perturbation bounds, as special cases, are also presented.
\end{abstract}

Mathematics subject classification (2010): 15A23, 15A45.

Keywords and phrases: Generalized Cholesky factorization, Cholesky-like factorization, multiplicative perturbation, rigorous perturbation bound, first-order perturbation bound.

\section{REFERENCES}

[1] P. Benner, R. Byers, H. Fassbender, V. Mehrmann, D. Watkins, Cholesky-like factorizations of skew-symmetric matrices, Electron. Trans. Numer. Anal. 11 (2000), 85-93.

[2] X. W. Chang, R.C. LI, Multiplicative perturbation analysis for QR factorizations, Numer. Algebra Control Optim. 1 (2011), 301-316.

[3] X. W. ChAng, D. STEhlÉ, Rigorous perturbation bounds of some matrices factorizations, SIAM J. Matrix Anal. Appl. 31 (2010), 2841-2859.

[4] X. W. Chang, C. C. Paige, G. W. Stewart, Perturbation analyses for the QR factorization, SIAM J. Matrix Anal. Appl. 18 (1997), 775-791.

[5] X. M. FANG, Multiplicative perturbation analysis for generalized Cholesky factorization, Numer. Math. J. Chinese Univ. 34 (2012), 61-68 (in Chinese).

[6] X. M. FANG, New perturbation analysis for the generalized Cholesky factorization, J. Zhanjiang Norm. College 30 (2009), 16-20 (in Chinese).

[7] G. H. Golub, C. F. Van Loan, Matrix Computation, third ed., The Johns Hopkins University Press, Baltimore, MD, 1996.

[8] H. B. GuO, W. G. WANG, Perturbation bounds for the Cholesky-like factorization of skew-symmetric matrix, Nanjing Univ. J. Math. Biquarterly 26 (2009), 43-50.

[9] REN-CANG LI, Relative perturbation bounds for positive polar factors of graded matrices, SIAM J. Matrix Anal. Appl. 25 (2005), 424-433.

[10] REN-CANG LI, Relative perturbation bounds for the unitary polar factor, BIT. 37 (1997), 67-75.

[11] REN-CANG LI, Relative perturbation theory: I. eigenvalue and singular value variations, SIAM J. Matrix Anal. Appl. 19 (1998), 956-982.

[12] REN-CANG LI, Relative perturbation theory: II. eigenspace and singular subspace variations, SIAM J. Matrix Anal. Appl. 20 (1999), 471-492.

[13] REN-CANG LI, G. W. STEWART, A new relative perturbation theorem for singular subspaces, Linear Algebra Appl. 313 (2000), 41-51.

[14] H. Y. LI, H. YANG, H. ShaO, A note on the perturbation analysis for the generalized Cholesky factorization, Appl. Math. Comput. 215 (2010), 4022-4027.

[15] K. VesElić, Perturbation theory for the eigenvalues of factorised symmetric matrices, Linear Algebra Appl. 309 (2000), 85-102. 
[16] W. G. WANG, Perturbation bounds for the generalized Cholesky factorization, Nanjing Univ. J. Math. Biquarterly 20 (2003), 113-119 (in Chinese).

[17] W. G. WANG, J. X. ZHAO, Perturbation analysis for the generalized Cholesky factorization, Appl. Math. Comput. 147 (2004), 601-606.

[18] G. W. Stewart, J. G. Sun, Matrix Perturbation Theory, Academic Press, Boston, 1990.

[19] J. X. ZHAO, The generalized Cholesky factorization method for saddle point problems, Appl. Math. Comput. 92 (1998), 49-58. 